\title{
Paris polyphylla ethanol extract induces G2/M arrest and suppresses migration and invasion in bladder cancer
}

\author{
Zhiyong Liu ${ }^{1}$, Zhonghua Sun ${ }^{2}$, Denglu Zhang ${ }^{1}$, Chenchen $\mathrm{Ma}^{1}$, Yuehua Jiang ${ }^{1}$, Guangshang Cao ${ }^{3}$, \\ Chao Sun ${ }^{4}$, Kailin $\mathrm{Li}^{4}$, Dawei $\mathrm{Xu}^{5}$, Jiang Liu ${ }^{6}$, Shengtian $\mathrm{Zhao}^{7}$
}

${ }^{1}$ Central Laboratory, ${ }^{2}$ Medical Department, ${ }^{3}$ Pharmacy Department, Affiliated Hospital of Shandong University of Traditional Chinese Medicine, Jinan, China; ${ }^{4}$ Department of Central Research Laboratory, The Second Hospital of Shandong University, Jinan, China; ${ }^{5}$ Department of Medicine, Division of Hematology and Centre for Molecular Medicine, Karolinska University Hospital Solna and Karolinska Institutet, Stockholm, Sweden; ${ }^{6}$ Internal Medicine of Traditional Chinese Medicine, Affiliated Hospital of Shandong University of Traditional Chinese Medicine, Jinan, China; ${ }^{7}$ Department of Urology, Shandong Provincial Hospital Affiliated to Shandong University, Jinan, China

Contributions: (I) Conception and design: S Zhao, J Liu, D Xu; (II) Administrative support: S Zhao, J Liu, Y Jiang; (III) Provision of study materials or patients: G Cao, C Sun; (IV) Collection and assembly of data: Z Liu, Z Sun, K Li; (V) Data analysis and interpretation: Z Liu, D Zhang, C Ma; (VI) Manuscript writing: All authors; (VII) Final approval of manuscript: All authors.

Correspondence to: Shengtian Zhao. Shandong Provincial Hospital Affiliated to Shandong University, No. 324, Jingwu Weiqi Road, Jinan, China. Email: zhaoshengtian@sdu.edu.cn; Jiang Liu. Affiliated Hospital of Shandong University of Traditional Chinese Medicine, No. 16369 Jingshi Road, Jinan, China. Email: sdzydfy@126.com; Dawei Xu. Department of Medicine, Division of Hematology and Centre for Molecular Medicine, Karolinska University Hospital Solna and Karolinska Institutet, Stockholm 17176, Sweden. Email: dawei.xu@ki.se.

Background: Paris polyphylla is a traditional Chinese medicinal herb with multiple antitumor activities, but the role of $P$. polyphylla in bladder cancer (BC) is under investigation. This study aims to examine the antitumor activities of $P$. polyphylla ethanol extract (PPE) on BC cells and elucidate the underlying mechanisms.

Methods: Viable cells were counted using the trypan blue exclusion assay. The cell cycle was analyzed using flow cytometry, and scratch wound-healing and transwell assays were used to evaluate cell migration and invasion abilities, respectively. The protein expression levels were determined by western blotting. A xenograft model was used to assess the in vivo inhibitory effect of PPE on BC tumor growth.

Results: Our results showed that PPE inhibited the growth of BC cells in vivo and in vitro. Mechanistically, PPE regulated the levels of cell cycle-associated proteins, with PPE-induced G2/M phase arrest occurring through cyclin-dependent kinase inhibitor 1 (CDKN1A) accumulation and cyclin B1 (CCNB1)/cyclindependent kinase 1 (CDK1) inhibition. BC tumor growth was also inhibited by PPE treatment. Moreover, the migration and invasion abilities of J82 cells were suppressed through modulating epithelial-mesenchymal transition (EMT) regulatory factors with upregulation of cadherin-1 (CDH1) and downregulation of cadherin-2 (CDH2), snail family transcriptional repressor 2 (SNAI2), and twist family bHLH transcription factor 1 (TWIST1).

Conclusions: PPE inhibited cell growth, induced G2/M arrest, and suppressed the migration and invasion of $\mathrm{J} 82$ cells. BC tumor growth in vivo was also inhibited by PPE. Our results lay the foundation for further studies on the antitumor mechanisms of PPE.

Keywords: Urinary bladder neoplasm; medicine, Chinese traditional; cell cycle; epithelial-mesenchymal transition (EMT)

Submitted Mar 15, 2020. Accepted for publication Aug 05, 2020.

doi: $10.21037 /$ tcr-20-1512

View this article at: http://dx.doi.org/10.21037/tcr-20-1512 


\section{Introduction}

Bladder cancer (BC) is a malignancy of the urinary system that is common in both China and western countries (1-3). The American Cancer Society has reported that BC is the fourth most common cancer in men with an estimated 62,100 new BC cases occurring in the United States in 2020 (4). Drug resistance is a major obstacle to BC chemotherapy, and more than fifty percent of superficial $\mathrm{BC}$ will recur in the future (5). Thus, BC patients need periodic urinary cytology and cystoscopy exams, which are costly and invasive. Because of this life-long recurrence monitoring, $\mathrm{BC}$ has become the most expensive disease among all cancers (6). Therefore, the identification of novel and effective drugs for BC treatment is urgently needed.

Paris polyphylla, a traditional Chinese medicinal herb distributed widely in Southwest China, might possess a broad spectrum of biological activities, including hemostatic, antimicrobial and antivenom activities, and it has been used for thousands of years (7-9). Clinical and pharmacological studies have shown that $P$. polyphylla has a hemostasis function via contraction of the uterus (10). The polyphyllins isolated from $P$. polyphylla possess antifungal activity against Cladosporium cladosporioides and Candida species (11). Polyphyllin I has been reported to possess antiinflammatory functions by inhibiting the phosphorylation of IKK $\alpha / \beta$ and $\mathrm{p} 65$, and suppressing the nuclear localization of p65 in the peritoneal macrophage-mediated inflammatory response (12). The components of $P$. polyphylla are used in more than 70 proprietary medicines, such as Gongxuening capsules, Chonglou jieduding tablets, and Jidesheng sheyao tablets (9).

$P$. polyphylla has also been used as a traditional Chinese medicine to treat cancers, including lung cancer, osteosarcoma, brain tumors, and other cancers (13). Polyphyllins, phytosterols and ecdysones are bioactive components that have been identified and isolated from P. polyphylla (7). Some of these chemical constituents have been demonstrated to possess antitumor activity. For instance, polyphyllin I induces apoptosis in gefitinibresistant non-small cell lung cancer via downregulating the MALAT1/STAT3 signaling pathway (14), caspase-3, -8 and -9 are activated and apoptosis is enhanced by polyphyllin $\mathrm{G}$ in human oral cancer cells (15). Additionally, invasion and epithelial-mesenchymal transition (EMT) are inhibited by polyphyllin I through the CIP2A/PP2A/ERK signaling pathway in prostate cancer (16). Additional antitumor activities of polyphyllins have also been found in gastric cancer (17) and glioblastoma (18); however, less is known about the antitumor effects of $P$. polyphylla on BC.

Abnormal growth and metastasis are the main characteristics of cancer. Cell mitosis is a precisely regulated process, and the cyclin B1 (CCNB1)/cyclin-dependent kinase 1 (CDK1) complex is a key regulator in G2 phase to M phase transition. Metastasis leads to approximately $90 \%$ of tumor-related deaths, and EMT is an essential process during tumor progression and metastasis. The activities of $P$. polyphylla toward BC have rarely been investigated. In this report, we studied the effects of $P$. polyphylla ethanol extract (PPE) on the cell growth, cell cycle, migration, invasion, and tumorigenic potential of BC cells. We present the following article in accordance with the ARRIVE reporting checklist (available at http://dx.doi.org/10.21037/tcr-201512).

\section{Methods}

\section{Extraction of P. polyphylla}

P. polyphylla was purchased from the Pharmacy Department, Affiliated Hospital of Shandong University of Traditional Chinese Medicine, and it was identified by Professor Xueshun Zhang. Two kilograms of P. polyphylla were ground into coarse powder, and a six-fold volume of EtOH/ $\mathrm{H}_{2} \mathrm{O}(60 / 40 \mathrm{v} / \mathrm{v})$ was added to reflux extraction two times, for 2 hours each. The extraction solution was combined and filtered, reducing the pressure of the filtrate to recover the ethanol and concentrate it to a relative density of approximately $1.10\left(60{ }^{\circ} \mathrm{C}\right)$. Two-fold volume of water was added and mixed, followed by incubation at $4{ }^{\circ} \mathrm{C}$ for 12 hours, and centrifugation at $6000 \mathrm{rpm}$ for 5 minutes, precipitation and drying under a reduced pressure (65$\left.70{ }^{\circ} \mathrm{C},-0.08 \mathrm{Mpa}\right)$ to collect the ground extract. The powder was dissolved in dimethyl sulfoxide (DMSO) to a final concentration of $1 \mathrm{mg} / \mathrm{mL}$.

\section{Cell culture}

The J82 BC-derived cell line (kindly provided by Associate Chief Technician Feng Kong, Shandong Provincial Hospital; RRID: CVCL_0359) was used in this present study. J82 cells were maintained in minimum essential medium (MEM; cat. no. CM10025; Macgene) supplemented with $10 \%(\mathrm{v} / \mathrm{v})$ fetal bovine serum (FBS; cat. no. 04-001-1ACS; Biological Industries). Cells were incubated in humidified incubators at $37{ }^{\circ} \mathrm{C}$ under an 
atmosphere with $5 \% \mathrm{CO}_{2}$.

\section{Trypan blue exclusion assay}

The test was performed using the Trypan Blue Staining Cell Viability Assay Kit (cat. no. KGY015; KeyGEN BioTECH) following the manufacturer's instructions. Briefly, J82 cells were treated with various concentrations of PPE for 24 hours and digested with trypsin. Cells were centrifuged at $300 \mathrm{~g}$ for five minutes after adding complete medium. The resulting cell pellet was washed once with PBS and resuspended in $100 \mu \mathrm{L}$ of PBS. The cell suspension $(90 \mu \mathrm{L})$ was gently mixed with $10 \mu \mathrm{L}$ of trypan blue solution, and allowed to stain for one minute, and the unstained cells were then counted using a blood cell count plate.

\section{CCK-8 assay}

The PPE concentrations used in the migration and invasion assay were analyzed using the Cell Counting Kit8 (CCK-8; cat. no. CK04; Dojindo). J82 cells were seeded in 96-well plates and cultured at $37{ }^{\circ} \mathrm{C}$ with $5 \% \mathrm{CO}_{2}$ for 24 hours, and various concentrations of PPE were added for 48 hours. After removing the medium, the J82 cells were incubated with new culture medium supplemented with $10 \%$ CCK- 8 for 2 hours. Absorption at $450 \mathrm{~nm}$ was detected using a microplate reader (Thermo Fisher Scientific).

\section{Colony formation assays}

J82 cells were seeded in 6-well plates (800 cells per well) and cultured with complete medium. The cells were then treated with various concentrations of PPE, and incubated at $37{ }^{\circ} \mathrm{C}$ for 10 days. The cells were then fixed with $4 \%$ paraformaldehyde for 15 minutes and stained with $0.2 \%$ crystal violet for 30 minutes. After washing with PBS for 5 minutes, colonies exceeding 50 cells were counted using Image-Pro Plus 6.0 (Media Cybernetics; RRID: SCR_007369).

\section{Scratch wound-bealing assay}

J82 cells were seeded in 6-well culture plates and grown to confluence overnight. Monolayer cells were scratched with a $200 \mu \mathrm{L}$ pipette tip, and the cells were washed at least three times with PBS to remove detached cells. Fresh medium with $1 \%$ FBS was added, and photographs were obtained at 0,24 , and 48 hours using a microscope (ZEISS). The cells were incubated in a humidified incubator at $37^{\circ} \mathrm{C}$ with $5 \%$ $\mathrm{CO}_{2}$.

\section{Transwell assay}

Transwell chambers (cat. no. 353097; Corning) were precoated with Matrigel (cat. no. 356234; BD) and incubated at $37{ }^{\circ} \mathrm{C}$ for 30 minutes. J82 cells were digested and resuspended in serum-free MEM. The cell suspension $\left(2.5 \times 10^{4} \mathrm{~J} 82\right.$ cells in $500 \mu \mathrm{L}$ of medium) was added to the upper wells, and the lower wells were filled with $600 \mu \mathrm{L}$ of MEM supplemented with $10 \%$ FBS. Subsequently, the chambers were incubated at $37^{\circ} \mathrm{C}$ for 48 hours, and the cells were then fixed with $4 \%$ paraformaldehyde for 15 minutes and stained with $0.1 \%$ crystal violet for 30 minutes. Cells in the upper chamber were gently wiped off with cotton swabs, and the invaded cells on the lower surface were counted under a microscope.

\section{Western blotting}

Whole cell protein lysates were obtained using RIPA buffer (cat. no. P0013E; Beyotime) supplemented with $1 \%$ phenylmethanesulfonyl fluoride (PMSF; cat. no. ST506; Beyotime), and protein concentrations were quantified using the BCA Protein Assay Kit (cat. no. P0012; Beyotime). Proteins $(40 \mu \mathrm{g})$ were separated on SDS-PAGE gels (cat. no. P0012A; Beyotime) and then transferred onto polyvinylidene fluoride (PVDF) membranes (cat. no. IPVH00010; Millipore). Membranes were blocked with 5\% nonfat milk and then incubated with the following primary antibodies: CCNB1 (cat. no. \#12231; RRID: AB_2783553) and CDK1 (cat. no. \#28439; RRID: AB_2798959) (purchased from Cell Signaling Technology); cyclindependent kinase inhibitor 1 (CDKN1A; cat. no. 103551-AP; RRID: AB_2077682) and cadherin-1 (CDH1; cat. no. 20874-1-AP; RRID: AB_10697811) (purchased from Proteintech Group Inc.); and cadherin-2 (CDH2; cat. no. ab98952; RRID: AB_10696943), snail family transcriptional repressor 2 (SNAI2; cat. no. ab27568; RRID: AB_777968) and twist family bHLH transcription factor 1 (TWIST1; cat. no. ab50581; RRID: AB_883292) (purchased from Abcam); all diluted 1:1,000. After incubation with secondary antibodies, a chemiluminescent horseradish peroxidase (HRP) substrate (cat. no. P90719; Millipore) was added, and the blots were imaged. 


\section{Flow cytometry}

After starvation overnight and treatment with various concentrations of PPE for 24 hours, J82 cells were collected and stained using a flow cytometry analysis kit (cat. no. GMS10021.1; GenMed Scientifics Inc.). The cell cycle was analyzed by flow cytometry (BD), and ModFit LT (Verity Software House; RRID: SCR_016106) was used to analyze the results.

\section{Xenograft studies}

All animal experiments were performed in compliance with the National Institutes of Health Guide for the Care and Use of Laboratory Animals, and were approved by the Animal Care and Use Committee of Shandong Provincial Hospital Affiliated to Shandong University (No.: 2019-005). Six-week-old male BALB/c nude mice were purchased from Vital River Laboratories (Beijing, China) and housed in separate cages in rooms with a controlled temperature (22 \pm $\left.2{ }^{\circ} \mathrm{C}\right)$, strict light-dark cycle (12:12) and airflow regulation, with free access to food and water throughout the study.

J82 cells $\left(5 \times 10^{6}\right)$ were injected subcutaneously into the right flanks of the mice. When the tumors were palpable $(2 \times 2 \mathrm{~mm})$, the mice were pair-matched by tumor size and divided into the following three groups: control group ( $0.9 \%$ sodium chloride, $\mathrm{n}=3)$, PPE $(100 \mathrm{mg} / \mathrm{kg})$ treatment group ( $\mathrm{n}=3)$, and $5-\mathrm{FU}(5 \mathrm{mg} / \mathrm{kg}$ ) treatment group (positive control, $n=3$ ). Intragastric administration (control and PPE treatment groups) and intraperitoneal injections (5FU treatment group) were performed daily, and the body weights and tumor sizes of the nude mice were measured every three days. After 21 days of treatment, the mice were sacrificed by cervical vertebra dislocation, and tumor tissues were collected for further analysis.

\section{Statistical analyses}

Data comparisons were conducted with one-way ANOVA, and the results are presented as means \pm SD. All statistical analyses were performed using GraphPad Prism 5.0 (GraphPad Software, Inc.; RRID: SCR_002798). Differences were considered to be significant when $\mathrm{P}<0.05$.

\section{Results}

\section{PPE inhibits the growth of 782 cells}

To evaluate the effect of PPE on BC, J82 cells were treated with various concentrations of $\operatorname{PPE}(0,1,3,9,27$, and $81 \mu \mathrm{g} / \mathrm{mL}$ ) for 24 hours. Cisplatin was used as a positive control, and viable cells were counted using the trypan blue exclusion assay. The results showed a dose-dependent decrease in the number of J82 cells after exposure to PPE. The $\mathrm{IC}_{50}$ value of PPE for J82 cells was $5.06 \pm 0.89 \mu \mathrm{g} / \mathrm{mL}$, which is comparable to that of cisplatin $\left(\mathrm{IC}_{50}=3.10 \pm\right.$ $0.77 \mu \mathrm{g} / \mathrm{mL}$ ) (Figure 1A). J82 cells were seeded into 6-well plates, and PPE was added as indicated. The imaging results showed that the morphology of cells in the PPE treatment and control groups was not obviously different. However, $3.2 \mu \mathrm{g} / \mathrm{mL}$ PPE notably inhibited J82 cell numbers after treatment for 24 hours (Figure 1B). Furthermore, the effect of PPE on the colony formation ability of J82 cells was assessed. The colony formation assay results showed that PPE significantly inhibited colony formation after treatment with PPE at $0.6,1.2$, and $1.8 \mu \mathrm{g} / \mathrm{mL}$ for 10 days (Figure 1C,D). These results indicated that PPE suppressed the growth of J82 cells in a dose-dependent manner. Furthermore, $3.2 \mu \mathrm{g} / \mathrm{mL}$ PPE was used as the highest concentration assayed in subsequent experiments.

\section{PPE induces G2/M phase arrest in 782 cells}

To assess the effect of PPE on cell cycle arrest in J82 cells, flow cytometry was used to analyze the cell cycle distribution. The results revealed an accumulation of cells in $\mathrm{G} 2 / \mathrm{M}$ phase after exposure to different concentrations of PPE, which gradually increased in a dose-dependent manner from $14.66 \%$ in the control group to $32.46 \%$ in the $3.2 \mu \mathrm{g} / \mathrm{mL}$ PPE treatment group (Figure $2 A$ ). Statistical analysis showed that the distribution of cells in G2/M phase arrest significantly increased after treatment with PPE at 1.6 and $3.2 \mu \mathrm{g} / \mathrm{mL}$ (Figure $2 B$ ). Taken together, these results suggested that PPE induced G2/M phase arrest in J82 cells.

\section{PPE inbibits xenograft tumor growth in vivo}

To investigate the effect of $\mathrm{PPE}$ on $\mathrm{BC}$ cells in vivo, a BC xenograft model was established as described in the Methods section. After 21 days of PPE treatment, mice were sacrificed, and tumor tissues obtained. Compared with the control group $(\mathrm{n}=3)$, the tumors from the 5-FU $(\mathrm{n}=3)$ and PPE $(\mathrm{n}=3)$ treatment groups were relatively small (Figure 3A). Furthermore, statistical analysis showed that both 5-FU and PPE significantly limited tumor weights (Figure 3B). The tumor growth curves for the control, PPE treatment, and 5-FU treatment groups are shown in 
A

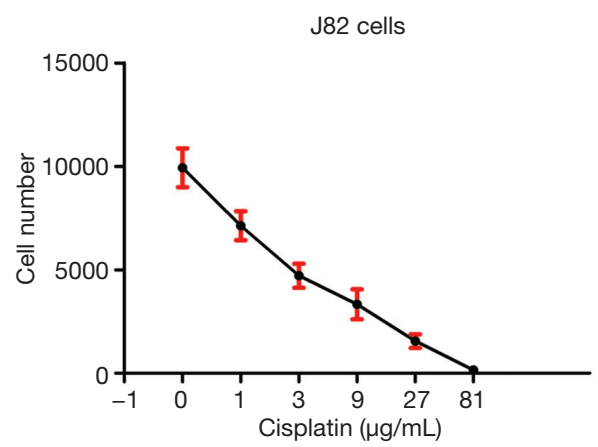

J82 cells

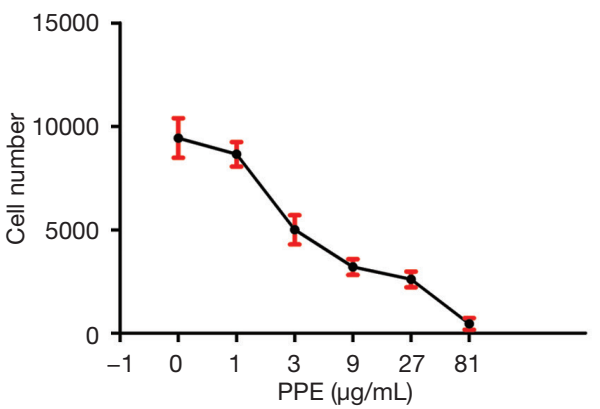

B

J82

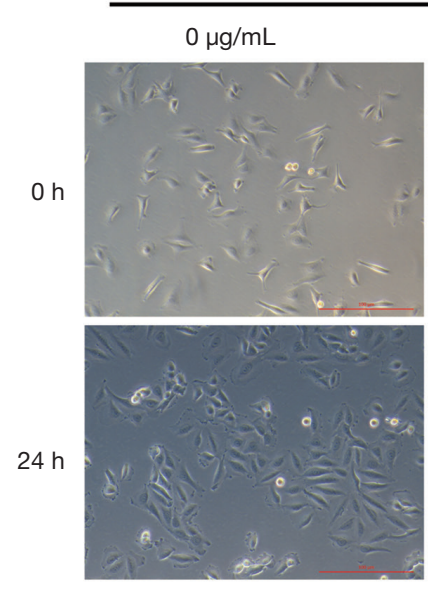

C

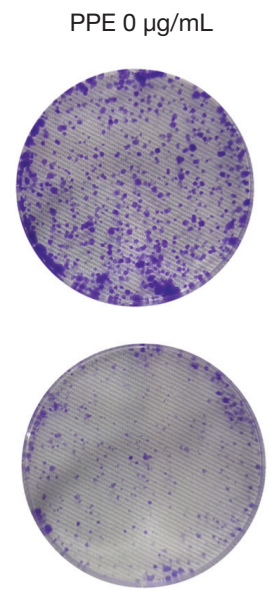

PPE $1.2 \mu \mathrm{g} / \mathrm{mL}$
PPE $0.6 \mu \mathrm{g} / \mathrm{mL}$
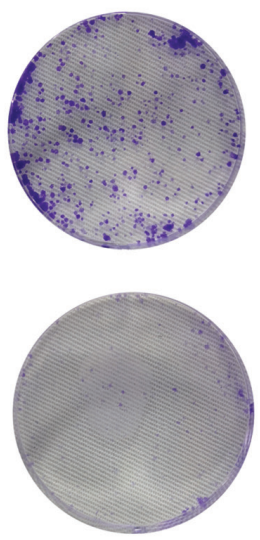

PPE $1.8 \mu \mathrm{g} / \mathrm{mL}$
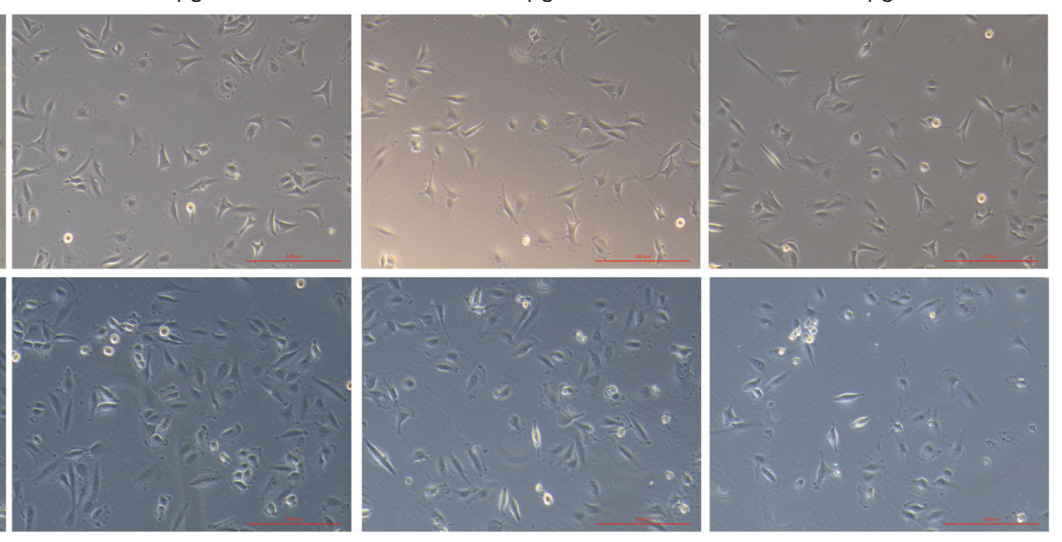

D

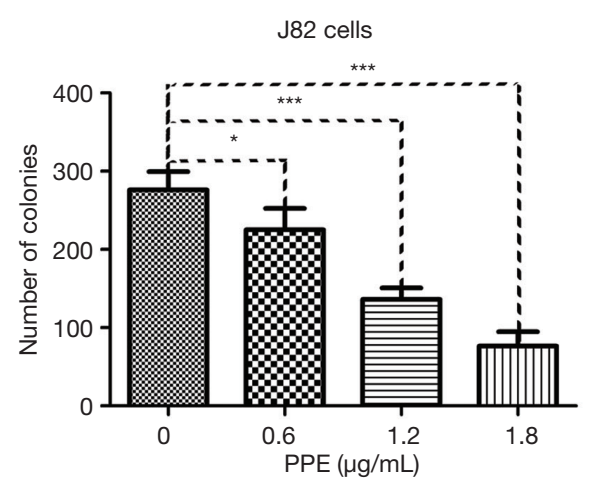

Figure 1 PPE inhibits J82 cell growth. (A) J82 cells were treated with PPE or cisplatin for 24 hours. Subsequently, viable cells were enumerated using the trypan blue exclusion assay. (B) Different concentrations of PPE were used to treat J82 cells for 24 hours. Cell morphologies and cell growth were recorded, and representative images are shown. Scale bar $=100 \mu \mathrm{m}$. (C) J82 cells were treated with PPE as indicated, and representative images of colony formation are shown. (D) The number of colonies was calculated and presented as the means $\pm \mathrm{SD}$ of three independent experiments $\left({ }^{*}\right.$ indicates $\mathrm{P}<0.05$; ${ }^{* * *}$ indicates $\left.\mathrm{P}<0.001\right)$. PPE, P. polyphylla ethanol extract. 

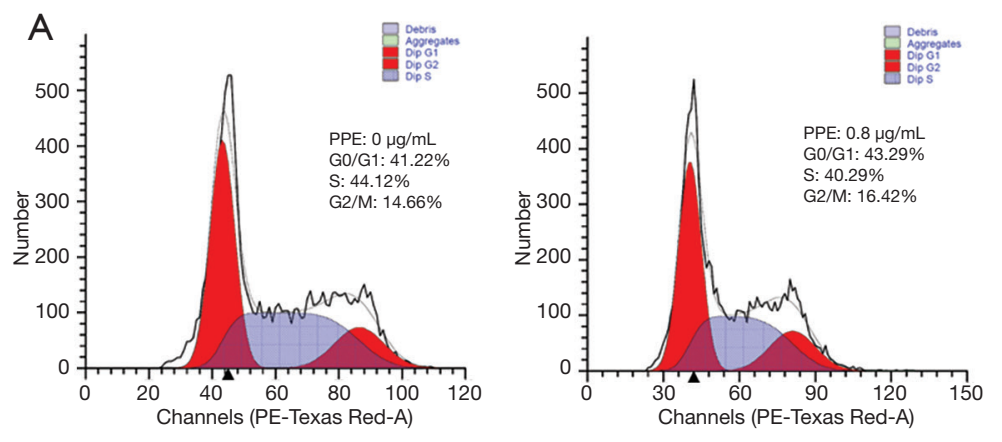

B
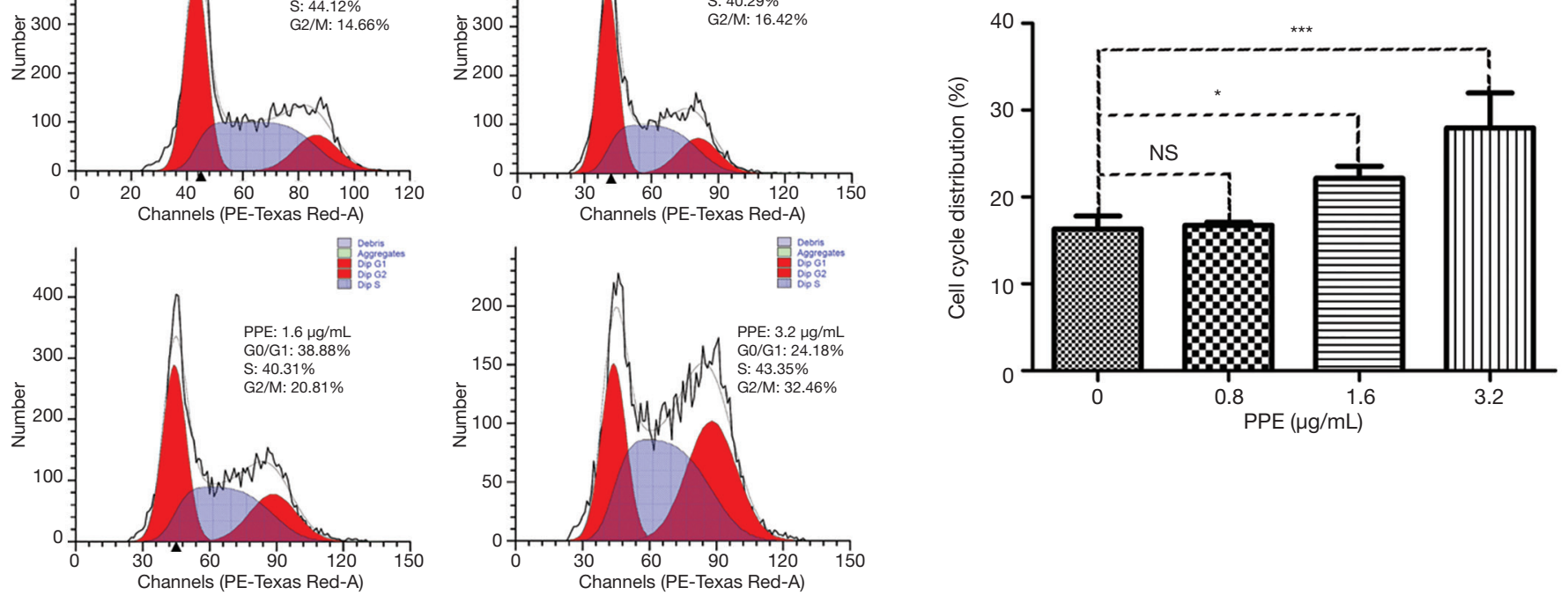

Figure 2 PPE induces G2/M phase arrest in J82 cells. J82 cells were seeded into 6-well plates and starved for 12 hours. Different concentrations of PPE (as indicated) were used to treat J82 cells for 24 hours, and cell cycle analyses were then performed. Cell cycle distributions are shown in (A and B). G2/M cell cycle arrest was statistically analyzed, and the results are presented as the means \pm SD of three independent experiments (NS represents not significant; * $\mathrm{P}<0.05 ;{ }^{* * *} \mathrm{P}<0.001$ ). PPE, P. polyphylla ethanol extract.

A

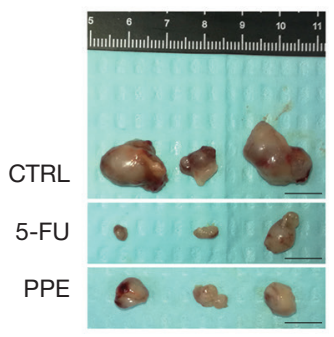

C

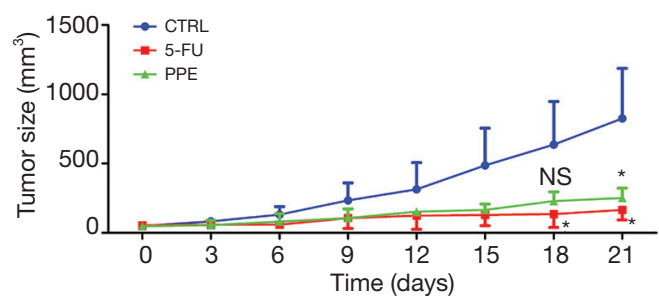

B

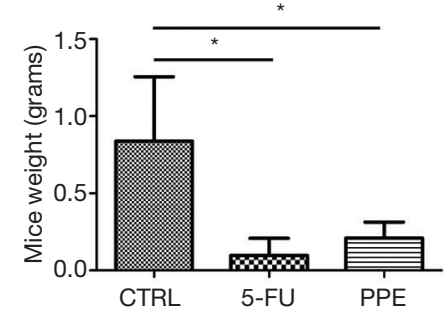

D

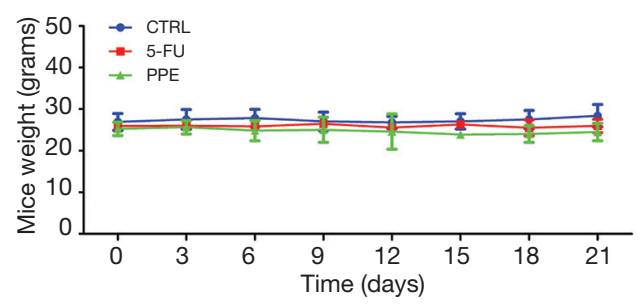

Figure 3 PPE inhibits xenograft tumor growth in vivo. (A) After 21 days of PPE treatment, the mice were sacrificed, and tumor tissues were obtained (scale bar $=1 \mathrm{~cm}$ ). (B) Tumor weights were calculated, and the results are presented as the means $\pm \mathrm{SD}$. The tumor sizes and weight curves of the three groups are shown in (C and D), respectively (NS represents not significant; ${ }^{*}$ indicates $\left.\mathrm{P}<0.05\right)$. PPE, $P$. polyphylla ethanol extract. 

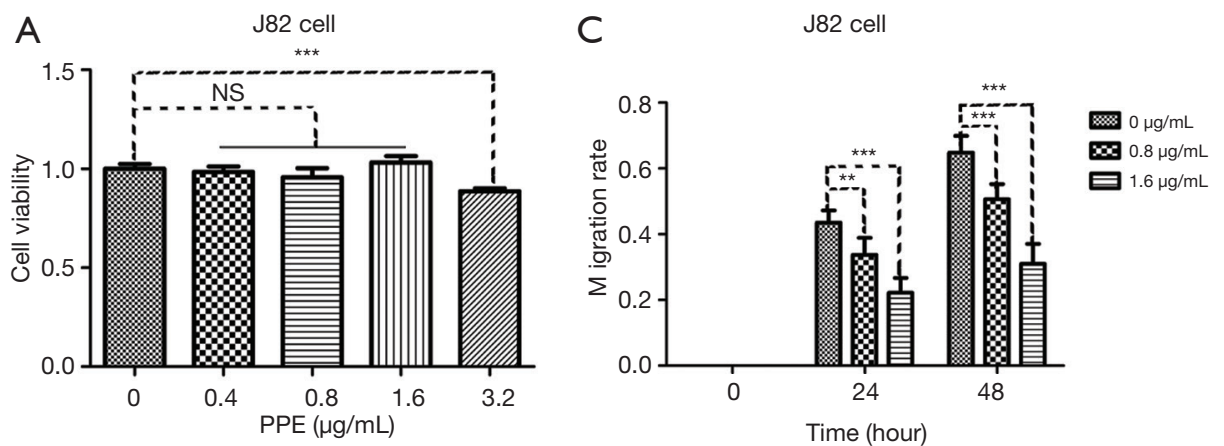

B

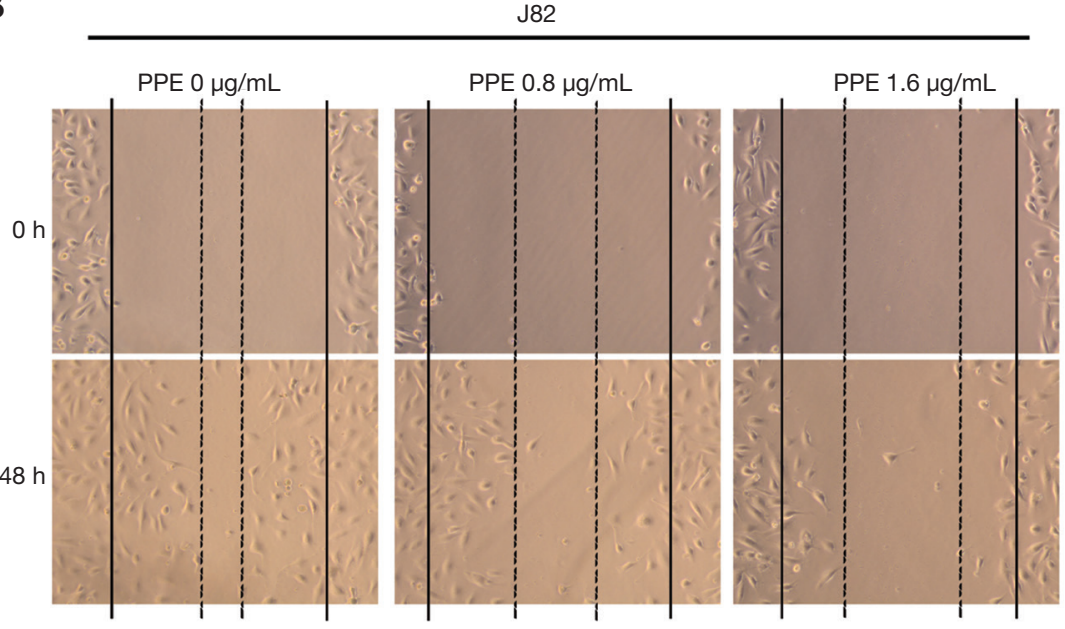

Figure 4 PPE inhibits J82 cell migration. (A) J82 cells were treated with PPE at the indicated concentrations for 48 hours, and a CCK-8 kit was used to evaluate cell viability. The error bars represent the SD (NS, not significant; *** $\mathrm{P}<0.001)$. (B) Confluent cells were scratched and then treated with various concentrations of PPE. Images show cells at 0 and 48 hours for each group (magnification, 100x). (C) Differences in migration rates between groups were evaluated (** indicates $\mathrm{P}<0.01$; ${ }^{* *}$ indicates $\mathrm{P}<0.001$ ). Data are representative of at least three experiments. PPE, P. polyphylla ethanol extract.

Figure 3C. Tumor volumes were significantly lower in the $\mathrm{PPE}$ and 5-FU treatment groups than the control group on days $21(\mathrm{P}<0.05)$. Moreover, the inhibitory activity of PPE with respect to tumor volume was comparable to that of 5-FU. The weights of the mice in the three groups are shown in Figure 3D. These results demonstrated that PPE inhibited xenograft tumor growth in vivo.

\section{PPE suppresses migration and invasion of $782 \mathrm{cells}$}

Migration and invasion are crucial processes in tumor cell metastasis. The CCK-8 assay was used to select PPE concentrations to be used in the migration and invasion assays, and this assay showed that cell viability was not affected by PPE at 0.8 and $1.6 \mu \mathrm{g} / \mathrm{mL}$ (Figure $4 A$ ). The effects of PPE on migration and invasion of $\mathrm{J} 82$ cells were determined by scratch wound-healing and transwell assays, respectively. According to the images and statistics, the cells treated with PPE $(0.8$ and $1.6 \mu \mathrm{g} / \mathrm{mL})$ for 48 hours showed significantly inhibited cell migration rates (Figure $4 B, C$ ) and numbers of invading cells (Figure $5 A, B$ ) compared with the control group $(0 \mu \mathrm{g} / \mathrm{mL})$. In addition, the inhibitory effect of PPE on migration and invasion was dose dependent.

\section{PPE modulates the levels of cell cycle-associated proteins and EMT-associated biomarkers in 782 cells}

To elucidate the molecular mechanisms underlying the growth inhibitory effects of PPE, J82 cells were starved overnight, and the levels of G2/M phase regulationassociated cytokines, including CDKN1A, CCNB1, and CDK1, were measured in $\mathrm{J} 82$ cells. The results revealed 
A

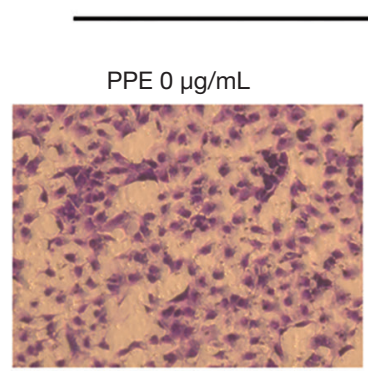

J82

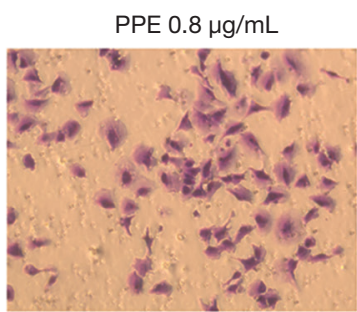

PPE $1.6 \mu \mathrm{g} / \mathrm{mL}$

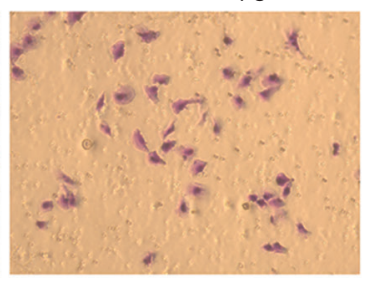

B J82 cells

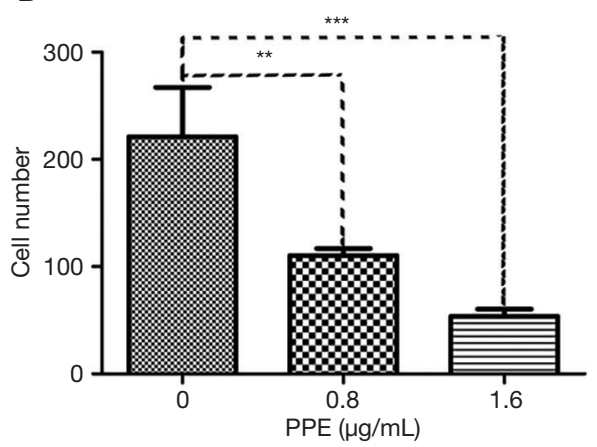

Figure 5 PPE inhibits J82 cell invasion. (A) J82 cells were treated with PPE at the indicated concentrations for 48 hours. Representative images show invaded cells in each PPE treatment group (magnification, 100x). (B) Differences in invading cell numbers between groups were analyzed (** indicates $\mathrm{P}<0.01$; ${ }^{* * *}$ indicates $\mathrm{P}<0.001$ ). Data are representative of three individual experiments. PPE, P. polyphylla ethanol extract.

A

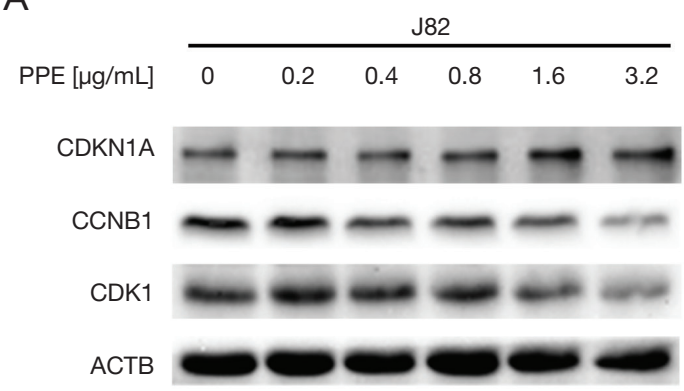

B $\mathrm{PPE}[\mu \mathrm{g} / \mathrm{mL}] \quad 0 \quad 0.4 \quad 0.8 \quad 1.6 \quad 3.2$

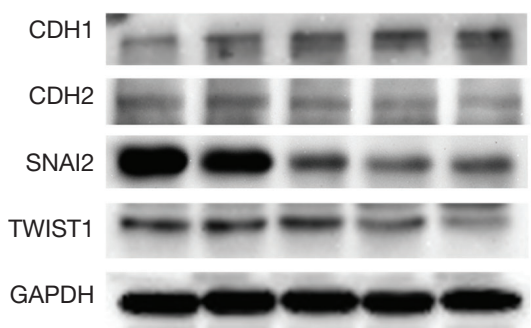

Figure 6 PPE modulates cell cycle signaling pathway and EMT-associated biomarkers. (A) J82 cells were starved for 12 hours. Subsequently, cells were treated with different concentrations of PPE for 24 hours, and the levels of cell cycle arrest-associated proteins were detected by western blotting. (B) J82 cells were treated with PPE at the indicated concentrations for 48 hours. Epithelial marker (CDH1), mesenchymal marker (CDH2), and transcription inhibitors of CDH1 (SNAI2 and TWIST1) were evaluated by western blotting. PPE, P. polyphylla ethanol extract; EMT, epithelial-mesenchymal transition.

that PPE treatment increased the level of CDKN1A, whereas it decreased those of CCNB1 and CDK1 (Figure 6A). These results indicated that PPE-induced G2/ $M$ phase arrest may occur via CDKN1A accumulation and inhibition of CCNB1/CDK1 expression.

EMT-associated biomarkers play essential roles in the process of metastasis. We analyzed whether PPE could repress $\mathrm{BC}$ cell invasion and migration through regulating the biomarkers. J82 cells were treated with PPE at concentrations of $0,0.4,0.8,1.6$, and $3.2 \mu \mathrm{g} / \mathrm{mL}$ for 48 hours, and EMT-related proteins were detected by western blot analysis. The results showed that the expression of CDH1 was increased after PPE treatment, but $\mathrm{CDH} 2$ expression was decreased. Moreover, the transcription inhibitors of CDH1 were also evaluated by western blot analysis, and the results showed that the expression levels of SNAI2 and TWIST1 were decreased (Figure 6B). These results indicated that PPE-induced inhibition of migration and invasion might occur through modulating EMT-associated biomarkers.

\section{Discussion}

$\mathrm{BC}$ is a common malignancy, and the cost of $\mathrm{BC}$ examination and treatment is high. Although resection, radical cystectomy, chemotherapy, and immunotherapy have been used to treat BC (19), drug resistance and recurrence remain unsolved issues. Therefore, the development of 
more effective drugs has been widely awaited. Antipyreticdetoxicate herbs are often used for cancer treatment in traditional Chinese medicine (20,21). As a heat-clearing and detoxifying herb, $P$. polyphylla shows potential antitumor properties $(22,23)$. Nevertheless, reports on the use of $P$. polyphylla to treat $\mathrm{BC}$ are rare. The results of the present study demonstrated that PPE has multiple antitumor functions in BC, including the inhibition of cell growth, induction of G2/M arrest and suppression of cell migration and invasion. BC cell growth in vivo is also inhibited by PPE.

The results of our previous study demonstrated that PPE could decrease the expression of mutant TP53 protein in $\mathrm{BC}$ cells (8). The results of the current study provide further insight into the mechanism of PPE activity against $\mathrm{BC}$, and we found that CCNB1 and CDK1 are downstream effectors of PPE-induced G2/M phase arrest. We further showed that PPE not only induces G2/M phase arrest but also suppresses the migration and invasion of $\mathrm{BC}$ cells. Moreover, we confirmed that PPE inhibits tumor cell growth in vivo.

Because sustained and proliferative signaling are hallmarks of cancer (24), targeting the aberrant expression of cell cycle regulators is a common cancer treatment strategy. CCNB1 and CDK1 are involved in regulating G2/ $M$ phase arrest, and CDKN1A is located upstream of these two regulators and inhibits their activity $(25,26)$. CDKN1A expression is promoted by TP53 accumulation, but TP53 is mutated in J82 cells (27). Although PPE may reduce mutant TP53 protein levels and increase CDKN1A levels, CDKN1A is also regulated by factors independent of TP53, including ER $\alpha$, STATs, and E2Fs (28). Thus, identification of the target sites of PPE and determination of whether it acts in a TP53-independent manner are important points that remain to be further investigated.

Tumor metastasis is the main reason cause of cancerrelated death (29), and it is an incurable disease. Therefore, the screening of anti-metastasis agents has become a priority. EMT is involved in tumor metastasis, and the vital characteristics of EMT are the upregulation of mesenchymal markers, such as $\mathrm{CDH} 2$, and downregulation of epithelial markers, including CDH1 (30). Traditional Chinese medicine provides a material and structural basis for drug screening. P. polyphylla possesses multiple biological activities, and PPE is an ethanol extract of $P$. polyphylla. Our results showed that PPE inhibits $\mathrm{BC}$ cell migration and invasion. We further showed that PPE upregulates CDH1 but downregulates CDH2, SNAI2, and TWIST1 protein expression levels. These findings indicated that PPE may be a potential candidate for anti-metastasis agents and merits further study.

Although natural and traditional herbs have some limitations, such as heterogeneity, methodological flaws and a limited regulatory framework, natural products have been an essential source of new drugs in the past nearly 40 years (31), and traditional Chinese medicine has been applied in anti-tumor treatments in the clinic (32-34). With quality control optimization and detailed mechanism of action elucidation, recognition of traditional Chinese medicine will be further improved.

Although our results showed that PPE could induce $\mathrm{G} 2 / \mathrm{M}$ arrest and inhibit the migration and invasion of $\mathrm{BC}$ cells, there is still a long way to go before its application in clinical treatment, including elucidation of the detailed molecular mechanism underlying the anti-tumor effect of PPE, whether the main polyphyllins possess the antitumor effect, and which polyphyllin exerts the most prominent antitumor activity against BC. Furthermore, oral or intravesical administration also merits further study. Nevertheless, we provide a potential candidate drug for further development in the treatment of BC.

\section{Conclusions}

The results of this study demonstrated that PPE has multiple antitumor functions toward BC, including the inhibition of cell growth, induction of G2/M arrest, and suppression of migration and invasion. BC cell growth in vivo was also inhibited by PPE. Thus, our results lay the foundation for further studies of the antitumor mechanisms of PPE.

\section{Acknowledgments}

We would like to thank Professor Xueshun Zhang (Affiliated Hospital of Shandong University of Traditional Chinese Medicine, Jinan, China) for identifying $P$. polyphylla herbs, and Associate Chief Technician Feng Kong (Shandong Provincial Hospital Affiliated to Shandong University, Jinan, China) for providing J82 cells.

Funding: This work was supported by the Key Clinical Projects of the Ministry of Health (Study on the risk factors for prostate cancer and establishment of screening model for high-risk population) [67], the Funds for the Disciplined Construction of High-Level Talents in the Affiliated Hospital of Shandong University of Traditional 
Chinese Medicine, and the Shandong Innovation Center for the National Human Genetic Resources sharing service platform [YCZYPT (2018)01-006].

\section{Footnote}

Reporting Checklist: The authors have completed the ARRIVE reporting checklist. Available at http://dx.doi. org/10.21037/tcr-20-1512

Data Sharing Statement: Available at http://dx.doi. org/10.21037/tcr-20-1512

Conflicts of Interest: All authors have completed the ICMJE uniform disclosure form (available at http://dx.doi. org/10.21037/tcr-20-1512). The authors have no conflicts of interest to declare.

Ethical Statement: The authors are accountable for all aspects of the work in ensuring that questions related to the accuracy or integrity of any part of the work are appropriately investigated and resolved. Experiments were performed under a project license (No.: 2019-005) granted by the Animal Care and Use Committee of Shandong Provincial Hospital Affiliated to Shandong University, in compliance with the National Institutes of Health Guide for the Care and Use of Laboratory Animals.

Open Access Statement: This is an Open Access article distributed in accordance with the Creative Commons Attribution-NonCommercial-NoDerivs 4.0 International License (CC BY-NC-ND 4.0), which permits the noncommercial replication and distribution of the article with the strict proviso that no changes or edits are made and the original work is properly cited (including links to both the formal publication through the relevant DOI and the license). See: https://creativecommons.org/licenses/by-nc-nd/4.0/.

\section{References}

1. Chen $\mathrm{W}$, Zheng R, Baade PD, et al. Cancer statistics in China, 2015. CA Cancer J Clin 2016;66:115-32.

2. Griffiths TR, Action on Bladder C. Current perspectives in bladder cancer management. Int J Clin Pract 2013;67:435-48.

3. Wang K, Liu T, Liu C, et al. TERT promoter mutations and TERT mRNA but not FGFR3 mutations are urinary biomarkers in Han Chinese patients with urothelial bladder cancer. Oncologist 2015;20:263-9.

4. American cancer society. Cancer facts \& figures 2020. Atlanta: American Cancer Society; 2020.

5. Hu H, Meng Q, Lei T, et al. Nucleophosmin1 associated with drug resistance and recurrence of bladder cancer. Clin Exp Med 2015;15:361-9.

6. Fankhauser CD, Mostafid H. Prevention of bladder cancer incidence and recurrence: nutrition and lifestyle. Curr Opin Urol 2018;28:88-92.

7. Shen S, Xu Z, Feng S, et al. Structural elucidation and antiaging activity of polysaccharide from Paris polyphylla leaves. Int J Biol Macromol 2018;107:1613-9.

8. Guo Y, Liu Z, Li K, et al. Paris Polyphylla-Derived Saponins Inhibit Growth of Bladder Cancer Cells by Inducing Mutant P53 Degradation While Up-Regulating CDKN1A Expression. Curr Urol 2018;11:131-8.

9. Qin XJ, Ni W, Chen CX, et al. Seeing the light: Shifting from wild rhizomes to extraction of active ingredients from above-ground parts of Paris polyphylla var. yunnanensis. J Ethnopharmacol 2018;224:134-9.

10. Tian Y, Zheng LH, Xu ZY, et al. Clinical and pharmacological study of the hemostatic action of rhizoma paridis by contraction of uterus. J Tradit Chin Med 1986;6:178-82.

11. Deng D, Lauren DR, Cooney JM, et al. Antifungal saponins from Paris polyphylla Smith. Planta Med 2008;74:1397-402.

12. Wang Q, Zhou X, Zhao Y, et al. Polyphyllin I Ameliorates Collagen-Induced Arthritis by Suppressing the Inflammation Response in Macrophages Through the NFkappaB Pathway. Front Immunol 2018;9:2091.

13. Wang YH, Shi M, Niu HM, et al. Substituting one Paris for another? In vitro cytotoxic and in vivo antitumor activities of Paris forrestii, a substitute of Paris polyphylla var. yunnanensis. J Ethnopharmacol 2018;218:45-50.

14. Yang Q, Chen $\mathrm{W}, \mathrm{Xu}$ Y, et al. Polyphyllin I modulates MALAT1/STAT3 signaling to induce apoptosis in gefitinib-resistant non-small cell lung cancer. Toxicol Appl Pharmacol 2018;356:1-7.

15. Hsieh MJ, Chien SY, Lin JT, et al. Polyphyllin G induces apoptosis and autophagy cell death in human oral cancer cells. Phytomedicine 2016;23:1545-54.

16. Liu X, Sun Z, Deng J, et al. Polyphyllin I inhibits invasion and epithelial-mesenchymal transition via CIP2A/ $\mathrm{PP} 2 \mathrm{~A} / \mathrm{ERK}$ signaling in prostate cancer. Int $\mathrm{J}$ Oncol 2018;53:1279-88.

17. He J, Yu S, Guo C, et al. Polyphyllin I induces autophagy and cell cycle arrest via inhibiting PDK1/Akt/mTOR 
signal and downregulating cyclin B1 in human gastric carcinoma HGC-27 cells. Biomed Pharmacother 2019;117:109189.

18. Liu J, Zhang Y, Chen L, et al. Polyphyllin I induces G2/M phase arrest and apoptosis in U251 human glioma cells via mitochondrial dysfunction and the JNK signaling pathway. Acta Biochim Biophys Sin (Shanghai) 2017;49:479-86.

19. Woolbright BL, Ayres M, Taylor JA 3rd. Metabolic changes in bladder cancer. Urol Oncol 2018;36:327-37.

20. Zhang L, Ren B, Zhang J, et al. Anti-tumor effect of Scutellaria barbata D. Don extracts on ovarian cancer and its phytochemicals characterisation. J Ethnopharmacol 2017;206:184-92.

21. Zhu H, Zhao H, Zhang L, et al. Dandelion root extract suppressed gastric cancer cells proliferation and migration through targeting lncRNA-CCAT1. Biomed Pharmacother 2017;93:1010-7.

22. Chang J, Wang H, Wang X, et al. Molecular mechanisms of Polyphyllin I-induced apoptosis and reversal of the epithelial-mesenchymal transition in human osteosarcoma cells. J Ethnopharmacol 2015;170:117-27.

23. Yao N, Ren K, Wang Y, et al. Paris polyphylla Suppresses Proliferation and Vasculogenic Mimicry of Human Osteosarcoma Cells and Inhibits Tumor Growth In Vivo. Am J Chin Med 2017;45:575-98.

24. Hanahan D, Weinberg RA. Hallmarks of cancer: the next generation. Cell 2011;144:646-74.

25. Jang SH, Kim AR, Park NH, et al. DRG2 Regulates G2/ M Progression via the Cyclin B1-Cdk1 Complex. Mol Cells 2016;39:699-704.

26. Czochor JR, Glazer PM. microRNAs in cancer cell response to ionizing radiation. Antioxid Redox Signal 2014;21:293-312.

27. Watanabe J, Nishiyama H, Matsui Y, et al. Dicoumarol

Cite this article as: Liu Z, Sun Z, Zhang D, Ma C, Jiang Y, Cao G, Sun C, Li K, Xu D, Liu J, Zhao S. Paris polyphylla ethanol extract induces G2/M arrest and suppresses migration and invasion in bladder cancer. Transl Cancer Res 2020;9(10):59946004. doi: $10.21037 /$ tcr-20-1512 potentiates cisplatin-induced apoptosis mediated by c-Jun $\mathrm{N}$-terminal kinase in p53 wild-type urogenital cancer cell lines. Oncogene 2006;25:2500-8.

28. Moussa RS, Park KC, Kovacevic Z, et al. Ironing out the role of the cyclin-dependent kinase inhibitor, p21 in cancer: Novel iron chelating agents to target p21 expression and activity. Free Radic Biol Med 2019;133:276-94.

29. Yan S, Zeng X, Tang Y, et al. Activating Antitumor Immunity and Antimetastatic Effect Through Polydopamine-Encapsulated Core-Shell Upconversion Nanoparticles. Adv Mater 2019;31:e1905825.

30. Loh CY, Chai JY, Tang TF, et al. The E-Cadherin and $\mathrm{N}$-Cadherin Switch in Epithelial-to-Mesenchymal Transition: Signaling, Therapeutic Implications, and Challenges. Cells 2019;8:1118.

31. Newman DJ, Cragg GM. Natural Products as Sources of New Drugs over the Nearly Four Decades from 01/1981 to 09/2019. J Nat Prod 2020;83:770-803.

32. Li CL, Hsia TC, Li CH, et al. Adjunctive Traditional Chinese Medicine Improves Survival in Patients With Advanced Lung Adenocarcinoma Treated With FirstLine Epidermal Growth Factor Receptor (EGFR) Tyrosine Kinase Inhibitors (TKIs): A Nationwide, Population-Based Cohort Study. Integr Cancer Ther 2019;18:1534735419827079.

33. Ma TT, Zhang GL, Dai CF, et al. Scutellaria barbata and Hedyotis diffusa herb pair for breast cancer treatment: Potential mechanism based on network pharmacology. J Ethnopharmacol 2020;259:112929.

34. Huang $\mathrm{Y}$, Zhu J, Lin X, et al. Potential of Fatty Oils from Traditional Chinese Medicine in Cancer Therapy: A Review for Phytochemical, Pharmacological and Clinical Studies. Am J Chin Med 2019;47:727-50. 